\title{
Documentos
}

\section{NEUROMODULACIÓN EN PATOLOGÍAS DE PISO PÉLVICO}

\author{
Valentín Manríquez G. ${ }^{1}$, César Sandoval S. ${ }^{1}$, Jorge Lecannelier A. ${ }^{1}$, Michel \\ Naser N. ${ }^{1}$, Rodrigo Guzmán R. ${ }^{1}$, Raúl Valdevenito S. ${ }^{1}$, Mario Abedrapo M. ${ }^{1}$ \\ 1 Unidad de Piso Pélvico Femenino, Hospital Clínico Universidad de Chile, Departamento de Obstetricia y Ginecología, \\ Facultad de Medicina, Universidad de Chile.
}

\section{RESUMEN}

A través de la historia la neuromodulación ha demostrado ser una alternativa de tratamiento eficaz en el manejo de diversas disfunciones del piso pélvico. Distintas técnicas intentan conseguir un objetivo común, sin embargo, el éxito terapéutico es disímil dependiendo de la severidad y tipo de patología. Describimos los aspectos clínicos y operacionales relacionados con las diversas técnicas, así como los mecanismos de acción propuestos para la neuromodulación.

\section{PALABRAS CLAVE: Piso pélvico, disfunción del tracto urinario inferior, incontinencia de orina, incontinencia fecal, neuromodulación}

\section{SUMMARY}

Through hystory, neuromodulation have proved to be an effective alternative of management of pelvic floor dysfunctions. Several technical alternatives try to reach a same therapeutic objetive, however, depending on the severity and type of symptom their succes differ. We describe the clinical and technical aspects related to those different technics so as the mechanisms of action that are propose for the neuromodulation.

\section{KEY WORDS: Pelvic floor, lower urinary tract dysfuntion, urine incontinence, fecal incontinence, neuromodulation}

\section{INTRODUCCIÓN}

Son pocas las alternativas de tratamiento, en la gama de enfermedades a la que nos enfrentamos como médicos, que son capaces de dar solución adecuada a un grupo importante de patologías utilizando siempre el mismo esquema terapéutico. Este es el caso de la neuromodulación, una herramienta que ha demostrado ser útil en tratar un número importante de disfunciones del piso pélvico (Tabla I). Sus orígenes se remontan a las décadas pasadas, cuando se intentaba tratar a pacientes con urgein- continencia refractarias a tratamiento médico y conductual con electroestimulación de la vejiga, raíces pundendas y sacras, para posteriormente evolucionar gracias al aporte de la acupuntura, con la estimulación de nervios periféricos en el tratamiento de afecciones vesicales.

El avance cualitativo en el desarrollo de nuevas y eficaces técnicas de estimulación se dio a mediados del siglo pasado, cuando se comenzó ha ensayar con la estimulación de raíces sacras en un intento por controlar los síntomas de pacientes con urgeincontinencia y dolor pélvico, idea llevada a 
cabo por Tanagho y cols (1), con el desarrollo de lo que hoy conocemos como neuromodulación sacra o central. El éxito de esta técnica, que ha sufrido numerosas variantes desde sus inicios, llevó a que finalmente la F.D.A. aprobara su uso en 1997 para el tratamiento de la urgeincontinencia. Actualmente existen series de grupos Norteamericanos y Europeos que dan cuenta de la efectividad de la técnica en el tratamiento de una amplia gama de disfunciones del piso pélvico $(2,3)$.

\section{Tabla I}

\section{APLICACIONES CLÍNICAS DE LA} NEUROMODULACIÓN

\begin{tabular}{ll}
\hline Esfera clínica & \multicolumn{1}{c}{ Síntoma } \\
\hline Patología urológica & $\begin{array}{l}\text { Vejiga hiperactiva } \\
\text { Retención urinaria no obstructiva } \\
\text { Síndrome doloroso vesical }\end{array}$ \\
Patología intestinal & $\begin{array}{l}\text { Incontinencia fecal } \\
\text { Constipación crónica }\end{array}$ \\
Partes blandas & $\begin{array}{l}\text { Dolor pélvico crónico } \\
\text { Dispareunia }\end{array}$ \\
\hline
\end{tabular}

En paralelo con el desarrollo de la neuromodulación central surgen en los Países Bajos y Norteamérica, grupos de trabajo que demostraron la utilidad de la estimulación eléctrica del nervio tibial posterior en pacientes con vejiga hiperactiva idiopática y cistitis intersticial $(4,5)$. Dando origen así a una nueva forma de realizar la neuromodulación que se conoce como neuromodulación periférica o tibial posterior. A esto se suma la publicación y desarrollo de la estimulación del nervio pudendo con electrodo implantable, que sin duda alguna se convierte también en otra alternativa eficaz (6).

En los últimos años y dada la demanda de los pacientes por métodos de tratamiento efectivos en enfermedades crónicas, que afecta en forma importante la calidad de vida, la neuromodulación ha irrumpido con fuerza y se ha posicionado en el mundo desarrollado, como una herramienta terapéutica que debe ser considerada y aprendida por quienes trabajen en la investigación y manejo de las patología ligadas al piso pélvico.

\section{MECANISMOS DE ACCIÓN}

Teoría de la compuerta. Originalmente descrita para explicar el alivio del dolor que ocasiona la estimulación eléctrica de una región corporal con la técnica TENS (estimulación eléctrica transcutánea), define la existencia de un circuito nervioso local entre el órgano a tratar y la raíz medular correspondiente, en el cual se distribuyen tres canales de estimulación, uno excitatorio, otro inhibitorio, y un tercero entre ambos que se activa o recluta luego de la estimulación eléctrica, y permite cerrar la compuerta a la vía del dolor y abrir la compuerta a la hipoalgesia (7). Este circuito local debe luego ocupar la vía aferente hacia el tronco encefálico y la corteza para lograr que, con la estimulación periódica el cambio perdure en el tiempo (8). Este podría corresponder al mecanismo de accion de la neuromodulacion en casos de dolor pélvico crónico e hiperactividad vesical.

Control supraespinal. La evidencia mas concreta del control supraespinal que circunda la fisiopatología de la neuromodulación esta en el trabajo de Finazzi-Agrò y cols (9) quienes demostraron en voluntarios portadores de vejiga hiperactiva, que al estimular una vía neurológica periférica en forma intermitente por un período de 12 semanas se logró evocar potenciales evocados somatosensoriales (PESS) a nivel frontal. Este hallazgo es sin duda de valiosa importancia, pues refleja la existencia de un cambio cortical generado a partir de la estimulación periférica. Esta neuroplasticidad evidenciada, es trascendental a la hora de entender el efecto terapéutico de largo plazo observado en pacientes tratados con neuromodulación periférica o central (Figura 1).

Quizás se puede realizar un simil con lo que ocurre al resetear un computador o al finalizar una sesión de electroshock cerebral, donde se permite una reorganización de la información reciente y la previamente conocida. Una vez lograda la modulación o reorganización de la información en el nivel cortical, el alivio del síntoma se hace conciente, y la

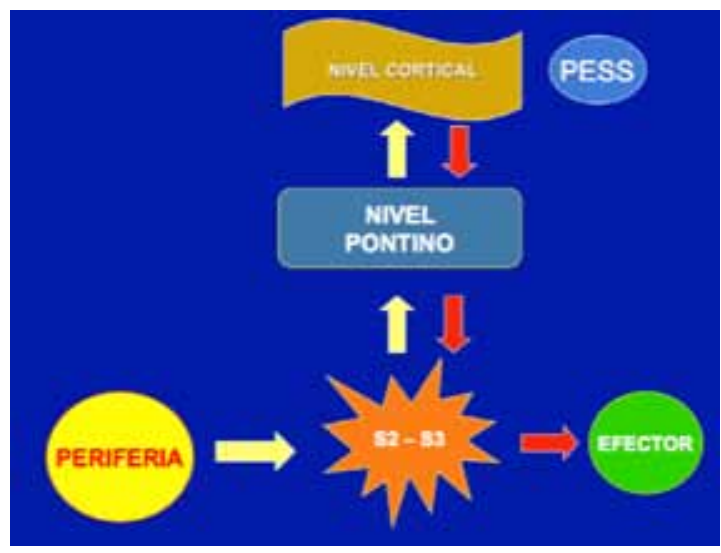

Figura 1. Esquema del mecanismo de acción de la neuromodulación central o periférica (PESS: potenciales evocados somatosensoriales). 
mejoría de la calidad de vida se hace cuantificable. Modulación del reflejo de guardia y reseteo "encendido - apagado". El reflejo de guardia corresponde a la respuesta uretral a la estimulación simpática. Corresponde a un reflejo excitatorio que ocasiona la contracción del músculo liso uretral durante la fase de llene vesical. Este reflejo es normalmente gatillado con los aumentos de presión intraabdominal de tal forma que contribuye a prevenir los escapes de orina de esfuerzo ante estornudos o tos. Se ha demostrado que este reflejo es también estimulado por terminaciones nerviosa vesicales que luego hacen sinapsis con interneuronas del plexo sacro la que se encargan de estimular directamente al nervio pudendo para que se genere la contracción del esfínter uretral (10). Es por este mecanismo que se puede lograr modular el reflejo de guardia al lograr la estimulación eléctrica aferente de esta vía, lo cual es de utilidad en casos de retención urinaria no obstructiva, en los cuales pueden coexistir la contracción no sinérgica del músculo detrusor y del esfínter uretral, generando así una retención urinaria no obstructiva.

Tanto o más importante que el reflejo de guardia es el reseteo del switch "encendido - apagado", responsable para muchos de la retención urinaria al existir un control anómalo del mismo a nivel central. El modo "encendido" implica espasticidad del piso pélvico y contracción de la musculatura uretral, mientras el modo "apagado" implica la relajación de los mismos, por lo tanto al lograr modular este reflejo y coordinarlo con la fase miccional, se puede lograr revertir la obstruccion urinaria antes desencadenada con la espasticidad de los grupos musculares descritos.

Estudios en voluntarios sanos con tomografía de emisión de positrones han demostrado que luego de la neuromodulación sacra se logran activar los centros pontinos y corticales relacionados con el control de la función vesical (8). Esta evidencia se suma a la descrita para los potenciales evocados somatosensoriales corticales que fueron desencadenados luego de la estimulación tibial posterior, lo cual sin duda confirma la teoría de que existe un control supraespinal que es fundamental para una adecuada respuesta terapéutica, y que luego de la estimulación repetida del vías neurológicas relacionadas, por algún mecanismo desconconocido, se orienta automáticamente hacia la solución correspondiente según cada caso.

\section{TÉCNICAS Y RESULTADOS}

Neuromodulación central: De las técnicas de neuromodulación que se describirán a continuación, el implante del Interstim (Medtronic, Mineapolis, Minnesota, USA) constituye aquel que requiere mayor capacitación por parte del operador para su instalación. Esta corresponde a una técnica que si bien, paso a paso, no representa un desafío mayor, incluye en ella una serie de conceptos de la neurofisiología y la neuroanatomía, de relevancia para el éxito del procedimiento.

Clásicamente se describe la realización del implante en dos tiempos:

1) Instalación de electrodo cuadripolar de prueba en tercer agujero sacro para testear la efectividad del procedimiento en relación con el alivio de los síntomas que motivaron su realización (urgeincontinencia, dolor pélvico crónico, incontinencia fecal). Se conecta este electrodo mediante un extensor a un neuroestimulador externo que la paciente porta durante el período de prueba, y si luego de un período de prueba que no debería exceder los 15 días se logró una mejoría cuantificada en al menos $50 \%$ respecto al basal, se procede con la segunda etapa.

2) Instalación del electrodo cuadripolar definitivo y conexión mediante un extensor a la batería definitiva, la cual se posiciona en un bolsillo supraaponeurótico en la región glútea superior, previamente tallado en la primera etapa del procedimiento. El tiempo de duración de la batería corresponde a 10 años para el modelo Interstim I actualmente disponible en nuestro país. Una vez terminado el procedimiento se realiza la programación de la batería por intermedio de un programador externo el cual configura la intensidad y los parámetros de estimulación que el tratante determine.

Ambas etapas del procedimiento se realizan con anestesia local y sedación superficial de la paciente, pues es necesario contar con su colaboración para identificar las sensaciones que se describen a nivel rectovaginal que indican el correcto posicionamiento del electrodo. En relación con esto, la adecuada ubicación del electrodo y la estimulación de este en el tercer agujero sacro, se confirma con la contracción del esfínter anal externo, que se manifiesta con la depresión de la región glútea inferior, la flexión plantar del ortejo mayor, y la percepción de parestesias en la zona rectovaginal referida por la paciente.

Es imprescindible para el procedimiento contar con el apoyo de un tecnólogo médico que maneje en forma adecuada el arco $\mathrm{C}$, pues su utilización resulta fundamental para la localización de los reparos anatómicos que ayudan en la instalación del electrodo en el lugar anatómico preciso (Figura 2). 

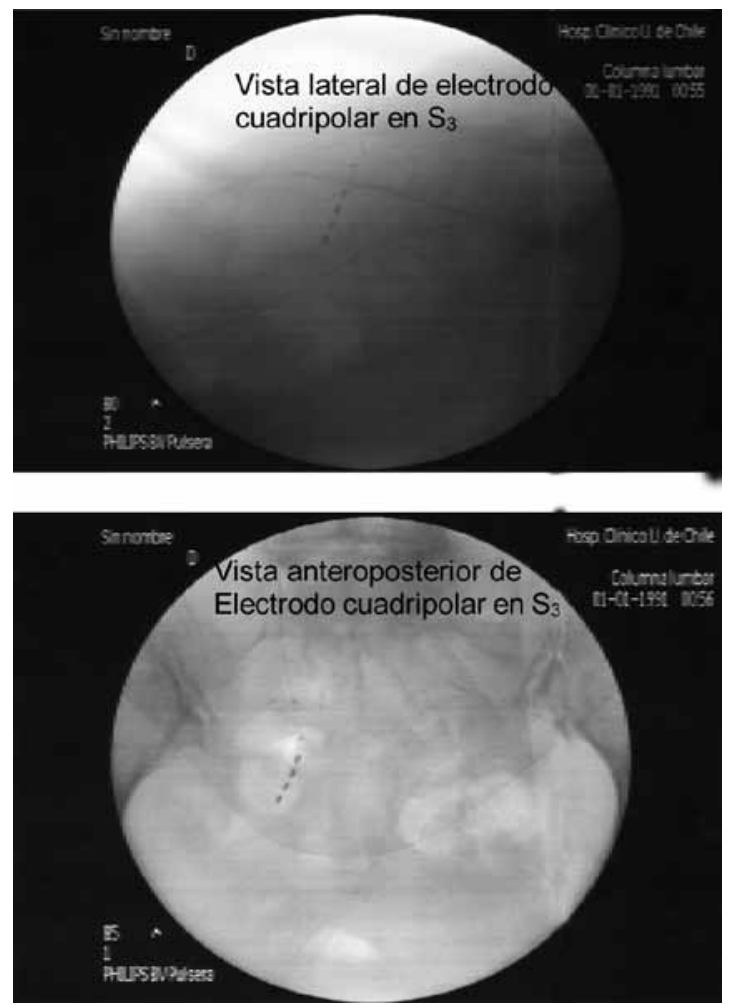

Figura 2. Visión radioscópica en la instalación del electrodo para estimulación sacra.

Respecto a la efectividad de la técnica en el tratamiento de los síntomas como la urgeincontinencia, la retención urinaria no obstructiva y el dolor pélvico crónico, los porcentajes de éxito varía dependiendo de las series analizadas. La experiencia norteamericana de 11 años en neuromodulación central publica con un seguimiento promedio de 22 meses que el éxito subjetivo mantenido es de $>50 \%$, > $80 \%$ y > $90 \%$ en el $70 \%, 50 \%$ y $35 \%$ respectivamente (3). Con mejoría significativa de la calidad de vida del $61 \%$ de los tratados. Por otro lado, la experiencia europea del grupo de Kessler, publicó un $100 \%$ de continencia en urgeincontinencia y reducción de 4 a 0 sondeos vesicales en pacientes con retención urinaria no obstructiva, con seguimiento promedio de 24 meses (2). En esta misma serie, los pacientes tratados por dolor pélvico crónico redujeron el puntaje de la escala visual análoga de 8 a 2 puntos al final del seguimiento.

Neuromodulación periférica: Definida como cualquier técnica de neuromodulación, que busque estimular en forma indirecta las raíces neurológicas responsables del funcionamiento motor y/o sensorial de algún órgano, víscera y/o estructura de so- porte. Actualmente en relación con las patologías de piso pélvico se describen principalmente la neuromodulación tibial posterior y la neuromodulación pudenda, que describimos a continuación.

a. Neuromodulación tibial posterior. En la búsqueda de una técnica de neuromodulación más simplificada y asequible nace la neuromodulación tibial posterior. Originaria de la medicina tradicional china, esta técnica ha evolucionado a través del tiempo y fue redescubierta por grupos Holandeses y Franceses quienes fueron los primeros en publicar el éxito de este tipo de estimulación en urgeincontiencia e incontinencia fecal respectivamente $(11,12,13)$. Para su desarrollo se utiliza originalmente la estimulación eléctrica del punto tibial posterior mediante un electrodo de aguja, y alternativamente un electrodo de superficie, y un electrodo de superficie que es el electrodo tierra, el cual se ubica a nivel del calcáneo. El punto tibial posterior fue descrito por la medicina tradicional china y se ubica 5 centímetros sobre el maleolo tibial por la cara interna de la pierna (Figura 3). Al ser este nervio una raíz del plexo sacro con origen en S2 - S4, su estimulación logra que en forma retrógrada se estimulen las raíces del plexo sacro que están relacionadas con el control visceral y muscular del piso pélvico. La clave para lograr una adecuada ubicación del electrodo activo es saber que el nervio a estimular es un nervio mixto, y que al estimular por sobre el umbral de lo sensitivo se produce una respuesta motora en el ortejo mayor sobre el cual ocurre una flexión plantar, marcando así la correcta ubicación de la aguja o el electrodo de superficie en el punto tibial posterior.

Cada sesión se prolonga por treinta minutos y se realiza según diferentes protocolos en forma semanal, bisemanal, trisemanal o incluso diaria $(14,15)$. Sin perjuicio de lo anterior, lo trascendente

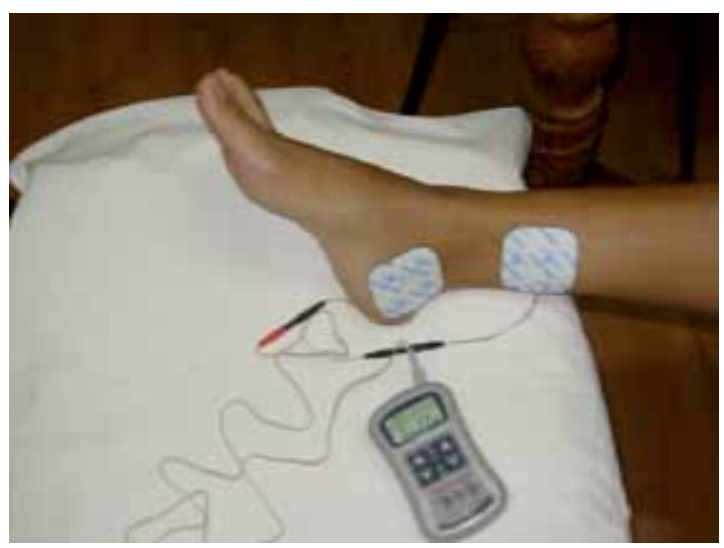

Figura 3. Instalación de dispositivo para neuromodulación tibial posterior transcutánea. 
es que el tiempo total de estimulación no sea inferior a 30 días en el caso de la estimulación diaria y no inferior a 6 semanas en el caso de las otras opciones con el fin de poder evaluar la respuesta inicial. Las características de la energía a utilizar se programan según los requerimientos del tratante, al igual que lo descrito para la neuromodulación central.

El éxito terapéutico descrito para esta técnica depende del síntoma a tratar y la severidad de este. En vejiga hiperactiva, es importante diferenciar si se trata de casos de pacientes con o sin urgeincontinencia y con o sin detrusor inestable. También es importante mencionar que es distinto referirse a la probabilidad de mejoría sintomática y a la probabilidad de continencia en el caso de pacientes con urgeincontinencia. En este respecto, se describe una probabilidad de respuesta terapéutica en pacientes con vejiga hiperactiva entre un $60-80 \%$, con una mejoría sintomática significativa respecto al placebo de $60 \%$ y una probabilidad de continencia de un $40 \%(11,12)$.

Con respecto a la incontinencia fecal y el dolor pélvico crónico, no existen datos tan contundentes como los mencionados anteriormente para la vejiga hiperactiva, sin embargo, se puede afirmar, que en incontinencia fecal se logra mejoría sintomática subjetiva entre 40 y $60 \%(13,15)$. Similar a lo publicado para los casos de dolor pélvico crónico (16).

b. Neuromodulación pudenda: Con la idea de buscar una alternativa de solución a aquellos pacientes en los cuales fracasaba la neuromodulacion central o sacra, se desarrolla la idea de poder realizar neuromodulación de las aferencias pudendas. Considerando que la neuromodulación central estimula en forma única la raíz $\mathrm{S}_{3}$ en la mayoría de los casos descritos, y que las aferencias pudendas en estudios de neurofisiología corresponden en un $4 \%$ a $S_{1}, 60 \% S_{2}$ y $35 \% S_{3}$, y además sabiendo que en el $18 \%$ de los sujetos la aferencia pudenda al plexo sacro es exclusiva de $\mathrm{S}_{2}$, es que probablemente este tipo de neuromodulación de solución a todos aquellos pacientes en los cuales la estimulación central de $S_{3}$ ha fracasado (6).

Los primeros resultados publicados corresponden a una serie de casos de pacientes con urgeincontinencia, tratados con la utilización del dispositivo llamado bion ${ }^{\circledR}$ (Advanced Bionics Corporation, Valencia, CA). Sus resultados con un $60 \%$ de continencia urinaria fueros asombrosos (6), sin embargo, por situaciones aún desconocidas para la mayoría de los lectores no fue sino hasta el año 2005 que volvió a resurgir la neuromodulación pudenda con la utilización del bion®. La experiencia acumulada con esta técnica es alentadora para quienes busca- mos la eficiencia terapéutica a un costo moderado para el usuario. Esta incorpora una test de prueba y luego el implante del bion® con un set de cirugía mínimamente invasiva, con la paciente sedada y la utilización de anestesia local, y la ayuda de la radioscopía como guía en el implante (Figura 4). Aunque preliminares los resultados de la experiencia publicada muestran una probabilidad de continencia en pacientes con vejiga hiperactiva entre 60 y $70 \%$, con mejoría singnificativa de la calidad de vida y demostración urodinámica asociada en relación con los parámetros de la curva volumen/ presión y flujo/presión (6).

Actualmente se encuentra en desarrollo la investigación referente a los resultados de la incontinencia fecal con la utilización de esta técnica, reportes de estos resultados en comunicaciones orales muestran su real beneficio y probablemente en el futuro asistamos a la consolidación de esta técnica como solución a una amplia gama de patologías de piso pélvico.

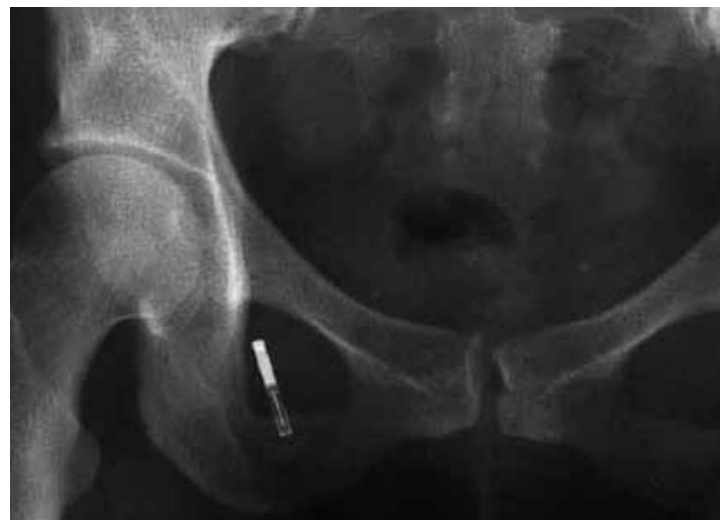

Figura 4. Representación radiográfica del implante para neuromodulación pudenda (con permiso de De la Portilla y cols.)

\section{COMENTARIOS}

Desde el año 2004 que nuestra Unidad se encuentra trabajando con la neuromodulación como una herramienta terapéutica más, útil en variadas disfunciones de piso pélvico. Es nuestra convicción con el ánimo de progresar como Unidad, Servicio, Hospital y Universidad, que es necesario que los grupos de investigación nacionales interesados en el tema, se involucren de manera multidisciplinaria y responsable, para lograr desarrollar este ámbito de la medicina con el éxito que conocemos en grupos extranjeros. Con este fin es fundamental homologar los instrumentos de evaluación objetiva y subjetiva, 
para así generar publicaciones de calidad que prestigien al medio. Es nuestro deseo incentivar el desarrollo de trabajos que no se limiten a la descripción de casos y el eventual éxito de una terapia novedosa, sino más bien apuntar la realización de la investigación prospectiva, que si bien demanda un mayor esfuerzo logístico, brinda la posibilidad de crecer y aportar información realmente útil a la comunidad médica y científica.

Actualmente en nuestra Unidad, se encuentra implementada la neuromodulación tibial posterior transcutánea, y hemos desarrollado con éxito los primeros casos de neuromodulación central, por lo que instamos a toda la comunidad médica interesada, a consultar sobre lo beneficios y las distintas modalidades de neuromodulación disponibles para la mejoría de la calidad de vida de los pacientes que lo requieran.

Por último, es necesario mencionar que todos los esfuerzos realizados por tratar disfunciones de piso pélvico redundan no tan solo en la mejoría de la calidad de vida del paciente, sino también en una considerable reducción de los gastos en los que incurre el paciente y su familia en el ánimo de tratar y paliar una enfermedad crónica. Se ha demostrado en pacientes con vejiga hiperactiva tratados con neuromodulación central, una reducción cercana al $90 \%$ del costo anual destinado a gastos médicos y domésticos relacionados con la enfermedad (17). Esto nos lleva a pensar que quizás más que un gasto, el valor cancelado por una determinada terapia se convierte en definitiva en una inversión costo efectiva para la salud familiar.

\section{BIBLIOGRAFÍA}

1. Tanagho EA, Schmidt RA. Electrical stimulation in the clinical management of the neurogenic bladder. J Urol 1988;140:1331-9.

2. Kessler T, Buchser E, Meyer S, Engeler D, Al-Khodairy $A$, Bersch $U$, et al. Sacral neuromodulation for refractory lower urinary tract dysfunction: results of a nationwide registry in Switzerland. Eur Urol 2007;51:1357-63.

3. Sutherland S, Lavers A, Carlson A, Holtz C, Kesha J, Siegel S. Sacral nerve stimulation for voiding dysfunction: one institution 11-year experience. Neurourol Urodyn 2007; 26:19-28.

4. McGuire EJ, Zhang SC, Horwinski ER, Lytton B.
Treatment of motor and sensory detrusor instability by electrical stimulation. J Urol 1983;129:78-9.

5. Stoller ML. Afferent nerve stimulation for pelvic floor dysfunction. Eur Urol 1999;35:132.

6. Groen J, Amiel C, Bosch R. Chronic pudendal nerve neuromodulation in women with idiopathic refractory detrusor overactivity incontinence: results of a pilot study with a novel minimally invasive implantable ministimulator. Neurourol Urodyn 2005;24:226-30.

7. van der Pal F, Heesakkers JP, Bemelmans BL. Current opinion on the working mechanisms of neuromodulation in the treatment of lower urinary tract dysfunction. Curr Opin Urol 2006;16:261-7.

8. Lazzeri M, Taddei I, Spinelli M. Sacral nerve modulation selectively increase regional cerebral blood flow in patients with chronic urinary retention. A preliminary SPET study. Eur Urol 2003;1(Suppl 2):169.

9. Finazzi-Agrò E, Rocchi C, Pachatz C, Petta F, Spera $\mathrm{E}$, Mori $\mathrm{F}$, et al. Percutaneous tibial nerve stimulation produces effects on brain activity: study on the modifications of the long latency somatosensory evoked potentials. Neurourol Urodyn 2009;28(4):320-4.

10. Alo KM, Holsheimer J. New trends in neuromodulation for the management of neuropatic pain. Neurosurgery 2002;50:690-703.

11. Vandoninck V, van Balken M, Finazzi Agrò E, Petta $\mathrm{F}$, Caltagirone $\mathrm{C}$, Heesakkers $\mathrm{J}$, et al. Posterior tibial nerve stimulation in the treatment of urge incontinence. Neurourol Urodyn 2003;22:17-23.

12. Vandoninck V, van Balken M, Finazzi Agrò E, Petta F, Micali F, Heesakkers J, et al. Percutaneous tibial nerve stimulation in the treatment of overactive bladder: urodynamic data. Neurourol Urodyn 2003;22:227-32.

13. Queralto M, Portier G, Cabarrot PH, Bonnaud G, Chotard JP, Nadrigny M, Lazorthes F. Preliminary results of peripherals transcutaneous neuromodulation in the treatment of idiopathic fecal incontinence. Int J Colorectal Dis 2006;21:670-2.

14. Finazzi Agrò E, Campagna A, Sciobica F, Petta F, Germani S, Zuccalà A, Miano R. Posterior tibial nerve stimulation: is the once a week protocol the best option? Minerva Urol Nefrol 2005;57:119-23.

15. Mentes BB, Yüksel O, Aydin A, Tezcaner T, Leventoğlu A, Aytaç B. Posterior tibial nerve stimulation for faecal incontinence after parcial spinal injury: preliminary report. Tech Coloproctol 2007;11:115-9.

16. Zhao J, Nordling J. Posterior tibial nerve stimulation in patients with intractable intersticial cystitis. BJU Int 2004;94:101-4.

17. Abosief S, Kim D, Rieder J, Rhee E, Meneffe S, Kaswick J, Ree M. Sacral neuromodulation: cost considerations and clinical benefits. Urology 2007;70:106973. 